\title{
Crizotinib-Associated Renal Cysts: A Case Series
}

\author{
Garima Suman ${ }^{1}$ Anurima Patra ${ }^{1}$ Amit Janu ${ }^{1}$ Akshay Baheti ${ }^{1}$ \\ ${ }^{1}$ Department of Radiology, Tata Memorial Center, Mumbai, \\ Maharashtra, India

\begin{abstract}
Address for correspondence Garima Suman, MD, Department of Radiology, Tata Memorial Center, Mumbai, Maharashtra, India
\end{abstract} \\ (e-mail: garimanmc@gmail.com).
}

J Gastrointestinal Abdominal Radiol ISGAR 2019;2:130-134

\begin{abstract}
Keywords

- crizotinib-associated renal cyst

- anaplastic lymphoma kinase

- nonsmall-cell lung cancer

- complex cyst

Crizotinib is an anaplastic lymphoma kinase (ALK) inhibitor, used as a targeted chemotherapeutic agent in ALK-positive cases of nonsmall cell lung carcinoma. Although uncommon, it may be associated with the formation of new renal cysts, which may be simple or complex, or the enlargement of preexisting simple renal cysts or their transformation into complex cysts. These cysts usually regress partially or completely but may rarely enlarge over time or get complicated by infection or abscess formation. Such cases may even require dose reduction or withholding crizotinib. Although documented in clinical literature, this entity is not well known among radiologists. Knowledge of this entity helps in preventing erroneous diagnosis of the new kidney "lesion" as metastasis or disease progression and avoids an unnecessary biopsy. We describe a series of four cases which developed complex renal cysts during treatment with crizotinib to demonstrate this point.
\end{abstract}

\section{Introduction}

Nonsmall-cell lung cancer (NSCLC) constitutes $85 \%$ of all lung cancers. ${ }^{1}$ Rearrangements of the anaplastic lymphoma kinase (ALK) gene are present in 3 to $5 \%$ of NSCLCs. ${ }^{2}$ Crizotinib is a first-generation orally active ALK inhibitor, which has revolutionized the treatment of ALK-positive NSCLC since receiving Food and Drug Administration approval in November 2013.

The development of renal cysts as an adverse effect of crizotinib has been described since clinical trials in 2011, with a reported incidence of 4 to $42 \% .^{3-7}$ With crizotinib assuming the center-stage in the treatment in NSLCs, the radiologist needs to be familiar with this entity so as to avoid misdiagnosing a new renal "lesion" as a metastasis. In this case series, we describe the imaging appearance and evolution of renal cysts in four cases of ALK-positive NSCLC treated with crizotinib at our institute.

\section{Case Reports}

1. A 64-year-old man was diagnosed with metastatic ALK-positive adenocarcinoma of the lung. He was started on crizotinib. In the baseline scan, both the kidneys were normal ( - Fig. 1A). Two months after initiation of crizotinib, the response assessment computed tomography
(CT) scan ( - Fig. 1B) revealed a new complex cystic lesion in right kidney, which almost doubled in size in next 3 months (-Fig. 1C). The patient's primary and metastatic disease otherwise responded well to the chemotherapy. This was correctly identified as a crizotinib-associated complex renal cyst and remained stable through the rest of therapy.

2. A 57-year-old woman with ALK-positive metastatic large cell neuroendocrine carcinoma was started on crizotinib. Response assessment CT scan done after 1 month ( -Fig. 2A) showed a new thick-walled cystic lesion in left kidney. This was labeled a crizotinib-associated renal cyst (CARC) and followed up. The renal lesion remained stable after the subsequent follow-up scans (Fig. 2B). There was partial response of the primary tumor.

3. A 40-year-old woman with ALK-positive metastatic NSCLC was started on crizotinib. Both kidneys were normal in the baseline scan. On follow-up CT after 2 months ( - Fig. $\mathbf{3 A 1}$ and A2), two new complex cystic lesions were seen, one each in either kidney.

After 4 more months, she developed flank pain and fever spikes. CT scan showed massive increase in the size of both the renal lesions with abscess formation and invasion into the renal bed ( $\boldsymbol{- \text { Fig. }}$ 3B). Both the lesions were drained

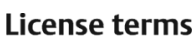 \\ ()(1) $\Theta \circledast$}



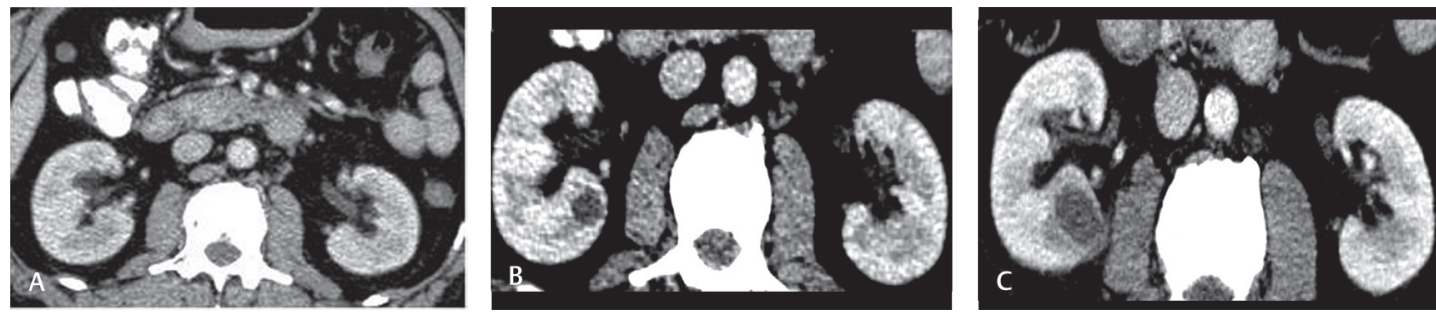

Fig. 1 (A) A 64-year-old patient with anaplastic lymphoma kinase-positive lung cancer. Axial baseline contrast-enhanced computed tomography (CECT) image shows normal kidneys. (B) Axial CECT image after 3 months of therapy shows a new simple cyst in the lower pole of right kidney. (C) Axial CECT image at 5 months follow-up scan shows interval increase in the size of right renal cyst, which now looks more complex with mild wall enhancement and mean Hounsfield unit 50 is seen (Bosniak IIF).
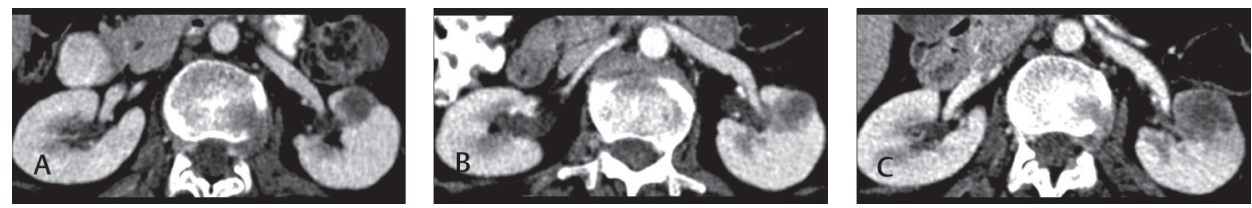

Fig. 2 (A) A 57-year-old woman with anaplastic lymphoma kinase-positive metastatic large cell neuroendocrine carcinoma. Baseline computed tomography (CT) (images not shown) demonstrated normal kidneys. Axial contrast-enhanced computed tomography (CECT) image after onemonth of initiation of crizotinib shows a hypodense lesion with thin imperceptible wall in theleft kidney (Bosniak I). (B) An 8-month follow-up axial CECT shows new thick peripheral wall enhancement with stable cyst size (Bosniak III cyst). (C) Further follow-up scan at 2 months interval showed mild increase in the size of left renal cyst, with persistent thick enhancing wall.
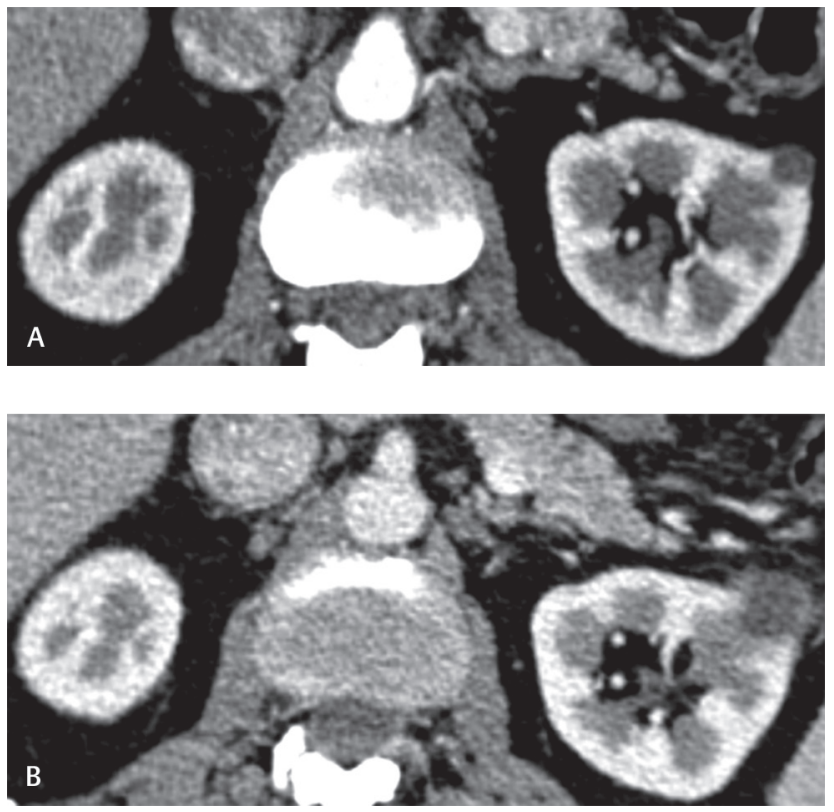

Fig. 3 (A) A 57-year-old man, diagnosed with anaplastic lymphoma kinase positive metastatic adenocarcinoma lung. Baseline scan demonstrated normal kidneys (not shown). Axial contrast-enhanced computed tomography (CECT) image after 3 months following initiation of therapy shows a new simple Bosniak I cyst in the left kidney. (B) CECT performed at 6 months follow-up shows increase in the size of the cyst with wall enhancement (Bosniak IIF cyst).

percutaneously. Aspirated fluid was negative for malignancy. The dose of crizotinib was halved and empirical broadspectrum antibiotics were started; however, the symptoms did not resolve. It was decided to withhold crizotinib in view of nonresolving infection.
Subsequent CT scan done after 2 months demonstrated near complete resolution of the bilateral renal lesions and perirenal inflammatory changes (Fig 3C). The patient was restarted on crizotinib after 3 more months at a lower dose. Unfortunately, this again led to an increase in the size of bilateral renal lesions and the formation of a large right iliopsoas abscess (Fig 3D). The abscess was drained by pigtail insertion and crizotinib was stopped till symptomatic improvement and then reinitiated. The patient is stable on follow-up since then.

4. A 57-year-old man, diagnosed with ALK-positive metastatic adenocarcinoma lung, was started on crizotinib in June 2017. The baseline CT showed unremarkable kidneys. On CT scan done after 3 months (September 2017), new simple cysts were seen in left kidney ( - Fig. 4A). On 6 months (December 2017) follow-up scan, the cysts showed increase in size ( - Fig. 4B). The patient was asymptomatic for any renal issues and is on routine follow-up.

\section{Scan Protocol}

Please note that these cysts were identified on routine follow-up CT done for these patients. CT scans were performed on 16 slice Siemens Somatom emotion CT scanner, manufactured in Erlangen, Germany. Following parameters were used to perform the contrast-enhanced CT scan: collimation, $4 \times 2.5 \mathrm{~mm}$; slice thickness, $1.5 \mathrm{~mm}$; reconstruction interval, 1.5 and $5 \mathrm{~mm}$; table feed, $12 \mathrm{~mm} /$ rotation. Kilovolt potential and milliampere seconds were adjusted in each case by using the automatic exposure control. Patients were given intravenous noniodinated contrast medium, either Ultravist (iopromide 300) or Omnipaque (iohexol 300) at a dose of $1.2 \mathrm{~mL} / \mathrm{kg}$ body weight with the scan performed at 70 seconds after the injection, using automatic pressure injector at a flow rate $2.8 \mathrm{~mL} / \mathrm{s}$. 

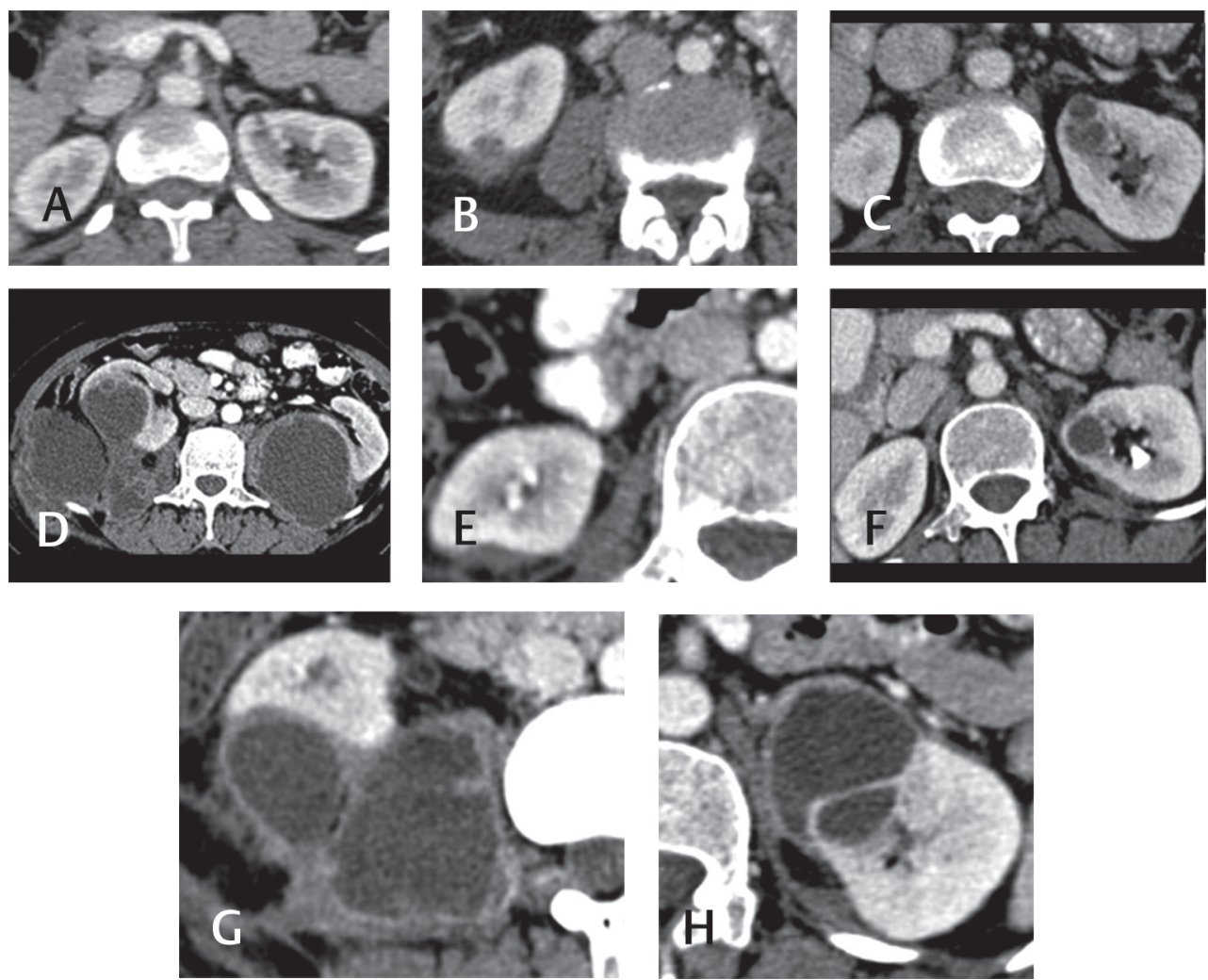

Fig. 4 (A) A 40-year-old woman with anaplastic lymphoma kinase-positive metastatic nonsmall cell carcinoma lung. Baseline axial computed tomography (CT) showed a tiny hypodensity in the left kidney, too small to characterize. The right kidney was normal. (B) Contrast-enhanced computed tomography (CECT) performed 3 months after starting crizotinib shows increased size of the left renal cyst without other worrisome features. (C) Another new exophytic complex cystic lesion is seen in the right renal upper pole. (D) Axial CECT image after another 3 months shows massive increase in size of both the renal cysts with abscess formation which invades the renal beds bilaterally. Aspiration showed suppurative inflammation. (E and F) Axial CECT image performed after withholding crizotinib for 2 months shows marked regression in the renal abscesses with a small residual component. ( $\mathbf{G}$ and $\mathbf{H}$ ) Axial CECT image performed after reinitiation of crizotinib shows a recurrent large septated iliopsoas collection and bilateral perinephric abscesses with thick enhancing walls. The right-sided collection was drained by pigtail insertion (not shown).

\section{Discussion}

The historical standard of care for treating NSCLCs has been platinum-based double agent chemotherapy. ${ }^{8}$ However, there has been paradigm shift in the treatment of NSCLC in the last decade, as a result of significant advances in molecular pathology and better understanding of heterogeneous tumor biology. Specific oncogenic driver mutations have been identified that lead to uncontrolled tumor proliferation, with epidermal growth factor receptor (EGFR) mutation being the most common, followed by ALK mutation. Targeted therapeutic agents have been developed to target these mutations by either small molecule inhibitors or receptor monoclonal antibodies. ${ }^{9}$ The successful targeted inhibition of these using EGFR tyrosine kinase inhibitors and crizotinib respectively has revolutionized the treatment of adenocarcinoma lung, with significantly improved progression free and overall survival in these patients. ${ }^{10}$

The association between crizotinib and development of renal cysts has been known since the drug was in phase III clinical trials. However, its pathogenesis is still not clearly understood. Tyrosine kinase receptor (c-mesenchymal to epithelial transition) is normally present in renal tubular epithelium and their inhibition by crizotinib could represent the responsible pathway. ${ }^{11}$
Crizotinib-associated cysts are usually simple but can be complex. They can present in the form of de novo appearance of new cysts, or as enlargement or increased complexity in preexisting cysts. The incidence of the various patterns of crizotinib-associated cysts as reported in literature is summarized in - Table 1. ${ }^{3-5,7,10}$ By far, the dominant pattern of evolution of CARCs is asymptomatic cysts that enlarge and spontaneously regress without the need for discontinuation of crizotinib or intervention. ${ }^{7,12}$ In our cases, the patients had stable cysts or progressively enlargement of cysts or transformation into complex cysts, without any impairment in renal function.

Table 1 Patterns of evolution crizotinib-associated renal cysts

\begin{tabular}{|l|l|}
\hline $\begin{array}{l}\text { Pattern of evolution of crizotinib-associated } \\
\text { renal cysts }\end{array}$ & Incidence (\%) \\
\hline Complex cysts $^{3-5,7}$ & $9-27$ \\
\hline Enlargement of cysts $^{3,4,7}$ & $2-32$ \\
\hline Septations & 25 \\
\hline Mixed cystic and solid appearance $^{7}$ & 29 \\
\hline Psoas/abdominal wall extension $^{5,7}$ & $7-41$ \\
\hline
\end{tabular}


Although ultrasonography (USG) can detect and characterize these cysts, CT usually forms the mainstay in characterization of these lesions, as it is anyway performed for response assessment in patients on crizotinib. On USG, CARCs may appear as ovoid, anechoic lesions with smooth well demarcated walls. Internal echoes and septations may be present in complex cysts and may sometimes cause a "pseudosolid" appearance. In such cases, posterior acoustic enhancement and lack of internal vascularity would favor complex cysts.

Similarly, CT may demonstrate a simple or complex cyst. It is important to obtain pre- and postcontrast images so that calcifications and hemorrhage can be accurately identified, and high-density nonenhancing renal cysts are not mistaken as solid metastases. Simple cysts are near water density with an upper threshold of 20 Hounsfield units (HU). ${ }^{13} \mathrm{Com}-$ plex features include hyperdense contents, septations, mixed solid cystic appearance, poorly defined margins, or extension into renal bed. The two commonest complex features in these CARCs are the development of multiple septations and a mixed cystic and solid appearance. The apparent solid appearance of some of these cysts may be due to the underlying inflammatory process. ${ }^{7}$ These cysts can fall into categories IIF, III, and IV of Bosniak classification..$^{14}$ It must be remembered that although Bosniak III/IV cysts usually need surgical resection, crizotinib-associated complex cysts are not known to have malignant potential and therefore resection is not recommended.

The differential diagnosis of renal cysts demonstrating complex features in a patient with NSCLC includes infections such as pyelonephritis and renal abscess, renal metastases, and primary renal malignancy. Infection can be excluded clinically based on lack of symptoms and urinalysis. Renal metastasis and primary renal neoplasm are rare; knowledge of CARC is, however, essential to avoid misinterpretation. Metastasis can have similar imaging morphology as the primary lesion in lung. ${ }^{15,16}$ Renal metastases have been described as small, multiple, bilateral, and located within the renal capsule. ${ }^{15,16}$ Delayed contrast-enhanced CT can help to distinguish renal cell carcinomas (RCC) and non-neoplastic cysts by measuring decrease in HU on delayed phase. RCC shows significant decrease in $\mathrm{HU}$ on delayed phase as opposed to benign cysts which remain unchanged. ${ }^{17} \mathrm{CT}$ and magnetic resonance imaging findings of complex cysts are similar; no significant difference is present between the two modalities in characterization of these lesions. ${ }^{14}$

Our case series highlights the importance of recognizing the imaging features of new renal cysts as an adverse effect of crizotinib treatment. Misinterpretation of such complex cysts as metastasis or disease progression may lead to futile invasive investigations, or worse, may lead to cessation of an otherwise effective chemotherapy. In cases in which complex renal cysts extend into adjacent extrarenal spaces, percutaneous drainage becomes necessary to hasten resolution. Existing case reports in literature and our own experience suggest that stopping crizotinib leads to their spontaneous regression. ${ }^{7,12}$

\section{Conclusion}

Development of renal cysts is not an uncommon side effect of treatment with crizotinib. Cysts are typically benign and remain stable on continued crizotinib therapy. They may, however, rarely enlarge over time or get complicated by infection or abscess formation. Such cases may even require dose reduction or withholding crizotinib. Complex cysts can mimic infection, primary renal malignancy or metastasis, or Bosniak IIF-IV category cysts. It is imperative that radiologists are aware of this entity in order to avoid wrong interpretation and mismanagement.

\section{Conflict of Interest}

None declared.

\section{References}

1 Molina JR, Yang P, Cassivi SD, Schild SE, Adjei AA. Non-small cell lung cancer: epidemiology, risk factors, treatment, and survivorship. Mayo Clin Proc 2008;83(5):584-594

2 Desai SS, Shah AS, Prabhash K, Jambhekar NA. A year of anaplastic large cell kinase testing for lung carcinoma: pathological and technical perspectives. Indian J Cancer 2013;50(2):80-86

3 Crizotinib Label FDA. Available at: http://www.accessdata.fda. gov/drugsatfda_docs/label/2013/202570s006lbl.pdf

4 Lin YT, Wang YF, Yang JC, et al. Development of renal cysts after crizotinib treatment in advanced ALK-positive non-small-cell lung cancer. J Thorac Oncol 2014;9(11):1720-1725

5 Schnell P, Bartlett $\mathrm{CH}$, Solomon BJ, et al. Complex renal cysts associated with crizotinib treatment. Cancer Med 2015;4(6):887-896

6 Yoneshima Y, Okamoto I, Arimura-Omori M, et al. Infected complex renal cysts during crizotinib therapy in a patient with non-small cell lung cancer positive for ALK rearrangement. Invest New Drugs 2015;33(2):510-512

7 Cameron LB, Jiang DHS, Moodie K, Mitchell C, Solomon B, Parameswaran BK. Crizotinib Associated Renal Cysts [CARCs]: incidence and patterns of evolution. Cancer Imaging 2017; 17(1):7

8 Schiller JH, Harrington D, Belani CP, et al; Eastern Cooperative Oncology Group. Comparison of four chemotherapy regimens for advanced non-small-cell lung cancer. N Engl J Med 2002;346(2):92-98

9 Chan BA, Hughes BGM. Targeted therapy for non-small cell lung cancer: current standards and the promise of the future. Transl Lung Cancer Res 2015;4(1):36-54

10 Solomon BJ, Mok T, Kim DW, et al. First-line crizotinib versus chemotherapy in ALK-positive lung cancer, for the PROFILE 1014 investigators. N Engl J Med 2014;371:2167-2177

11 Horie S, Higashihara E, Nutahara K, et al. Mediation of renal cyst formation by hepatocyte growth factor. Lancet 1994;344(8925):789-791

12 Klempner SJ, Aubin G, Dash A, Ou SH. Spontaneous regression of crizotinib-associated complex renal cysts during continuous crizotinib treatment. Oncologist 2014;19(9):1008-1010

13 Jia JB, Lall C, Tirkes T, Gulati R, Lamba R, Goodwin SC. Chemotherapy-related complications in the kidneys and collecting system: an imaging perspective. Insights Imaging 2015;6(4):479-487

14 Israel GM, Bosniak MA. How I do it: evaluating renal masses. Radiology 2005;236(2):441-450 
15 Scatena C, Comin CE, Lapini A, Raspollini MR. Renal metastasis from pulmonary adenocarcinoma: the pathologist's approach to an uncommon finding: case report and review of the literature. Appl Immunohistochem Mol Morphol 2013;21(5):460-4631

16 Honda H, Coffman CE, Berbaum KS, Barloon TJ, Masuda K. CT analysis of metastatic neoplasms of the kidney.
Comparison with primary renal cell carcinoma. Acta Radiol 1992;33(1):39-44

17 Voci SL, Gottlieb RH, Fultz PJ, et al. Delayed computed tomographic characterization of renal masses: preliminary experience. Abdom Imaging 2000;25(3):317-321 